

\title{
La spesa pubblica bolognese alla fine del XIII secolo. Prime indagini sul Liber expensarum del 1288

\author{
Marco Conti
}

\section{To cite this version:}

Marco Conti. La spesa pubblica bolognese alla fine del XIII secolo. Prime indagini sul Liber expensarum del 1288. Mélanges de l'École française de Rome - Moyen Âge, 2016, 128 (2), 10.4000/mefrm.3329 . hal-01367670

\section{HAL Id: hal-01367670 \\ https://hal.science/hal-01367670}

Submitted on 16 Sep 2016

HAL is a multi-disciplinary open access archive for the deposit and dissemination of scientific research documents, whether they are published or not. The documents may come from teaching and research institutions in France or abroad, or from public or private research centers.
L'archive ouverte pluridisciplinaire HAL, est destinée au dépôt et à la diffusion de documents scientifiques de niveau recherche, publiés ou non, émanant des établissements d'enseignement et de recherche français ou étrangers, des laboratoires publics ou privés. 
Mélanges

de l'École française de Rome

Moyen Âge

Mélanges de l'École

française de Rome - Moyen

Âge

128-2 (2016)

Dossier : « Ben più del denaro ». Il diritto della moneta, la sua sovranità, le sue funzioni Varia - Atelier doctoral - Regards croisés

Marco Conti

\title{
La spesa pubblica bolognese alla fine del XIII secolo. Prime indagini sul Liber expensarum del 1288
}

\begin{abstract}
Avertissement
Le contenu de ce site relève de la législation française sur la propriété intellectuelle et est la propriété exclusive de l'éditeur.

Les œuvres figurant sur ce site peuvent être consultées et reproduites sur un support papier ou numérique sous réserve qu'elles soient strictement réservées à un usage soit personnel, soit scientifique ou pédagogique excluant toute exploitation commerciale. La reproduction devra obligatoirement mentionner l'éditeur, le nom de la revue, l'auteur et la référence du document.

Toute autre reproduction est interdite sauf accord préalable de l'éditeur, en dehors des cas prévus par la législation en vigueur en France.
\end{abstract}

\section{revues.org}

Revues.org est un portail de revues en sciences humaines et sociales développé par le Cléo, Centre pour l'édition électronique ouverte (CNRS, EHESS, UP, UAPV).

Référence électronique

Marco Conti, «La spesa pubblica bolognese alla fine del XIII secolo. Prime indagini sul Liber expensarum del 1288 », Mélanges de l'École française de Rome - Moyen Âge [En ligne], 128-2 | 2016, mis en ligne le 06 septembre 2016, consulté le 07 septembre 2016. URL : http://mefrm.revues.org/3329

Éditeur : École française de Rome

http://mefrm.revues.org

http://www.revues.org

Document accessible en ligne sur :

http://mefrm.revues.org/3329

Document généré automatiquement le 07 septembre 2016.

(c) École française de Rome 


\section{Marco Conti}

\section{La spesa pubblica bolognese alla fine del XIII secolo. Prime indagini sul Liber expensarum del 1288}

La documentazione fiscale della città di Bologna è stata oggetto delle prime ricerche a partire dagli anni trenta del secolo scorso. In questo primo periodo, l'interesse si è concentrato sulla decima pagata dal comune (lavoro che faceva parte del progetto sulle Rationes decimarum italiae) e sulle dichiarazioni d'estimo di Pier de Crescenzi, usate allo scopo di conoscere il patrimonio dell'agronomo bolognese ${ }^{1}$. Negli anni Cinquanta, Gianfranco Orlandelli cominciò a studiare le fonti finanziarie del comune in modo più sistematico, mettendo in luce la questione della revisione del bilancio ${ }^{2}$. Poi negli anni Settanta e Ottanta, gli studi si sono moltiplicati prendendo in esame principalmente le fonti che costituiscono il sistema delle imposte dirette e indirette ${ }^{3}$. Più recentemente infine si è messo in evidenza la prassi amministrativa dell'elaborazione dell'estimo, il tema dell'evasione fiscale, quello del debito pubblico e gli inventari dei beni comunali ${ }^{4}$.

A differenza di quanto è avvenuto con le entrate, lo studio delle spese del comune è stato piuttosto scarso. Si tratta invece di una questione altrettanto se non più importante, dato che, come è stato dimostrato negli ultimi anni dagli studi di Maria Ginatempo, nei comuni erano i bisogni e le necessità a determinare il drenaggio delle risorse e non il contrario 5 . La spesa pubblica costituisce dunque un mezzo privilegiato per comprendere il tipo di politica fiscale adottata dal comune. Questo scritto ha per obiettivo il primo sondaggio di un documento particolarmente utile al fine sopraindicato : il liber expensarum del 1288 conservato all'Archivio di Stato di Bologna ${ }^{6}$.

Nella prima parte dell'articolo, il liber sarà studiato nelle sua struttura intrinseca ed estrinseca e messo in relazione con le altre scritture dello stesso ufficio cittadino per comprenderne il ruolo. Nella seconda parte saranno analizzate con precisione le quantità di denaro destinate alle varie voci della spesa pubblica, in modo da mettere in evidenza le scelte di politica fiscale intraprese dal comune?

\section{Liber expensarum}

\section{Libri contabili}

In questa sede non è possibile presentare tutti i tipi di documentazione amministrativa e finanziaria prodotti dal comune. Ci limiteremo che ad una rapida introduzione delle scritture contabili della fine del XIII secolo, conservate nell'Archivio di Stato di Bologna.

I registri contabili superstiti per questo periodo, oltre al liber oggetto del nostro studio, sono stati prodotti da due uffici cittadini : il disco dell'orso (ufficio che si occupava delle entrate inerenti le multe, le condanne e le collette) e l'officium bladi (che si occupava dell'acquisto e vendita del grano e altri cereali). Per il disco dell'orso si tratta di tre frammenti di libri introituum, mentre per l'officium bladi, sono conservati un liber totius introytus proventi datio bladi e due libri introituum et expensarum ${ }^{8}$. Una rapida analisi di questi registri, ci permette di comprendere che questi uffici funzionavano come delle casse autonome, gestite separatamente dalla tesoreria cittadina. A Bologna, come in altri comuni, per esempio a Siena o Cremona, la tesoreria cittadina era solo uno degli uffici che gestiva il denaro del comune e ciascuno doveva produrre dei registri contabili, relativi alle proprie entrate e uscite ${ }^{9}$.

Tutti i registri venivano sottoposti al controllo da parte del giudice al sindacato e in seguito archiviati nella camera actorum del comune ${ }^{10}$. Il liber expensarum del 1288 fa dunque parte di un complesso di manoscritti contabili molto ampio. Le voci di spesa che saranno presentate in seguito fanno riferimento all'attività della tesoreria e non al complesso della contabilità cittadina. 
Per comprendere l'utilizzo del liber expensarum del 1288 all'interno della tesoreria e all'interno del panorama documentario della città è necessario metterlo in relazione con gli altri registri e frammenti di registri di entrate e uscite prodotti dalla tesoreria bolognese dalla fine del XIII secolo all'inizio del XIV secolo. Il liber introituum del 1298 è composto da più quaterni in pergamena, ciascuno dei quali identificato da una segnatura numerica (per esempio tercius quaternus .III.) e dalla data. Di seguito, sono indicate in ordine cronologico tutte le entrate riguardanti l'officium gabelle e i massari del contado.

Sull'ultima carta è riportata l'indicazione : «Liber [...] continentur in se treginta quatuor cartas inter scriptas et non scriptas, viginti octo et due dimidie sunt scripte et alienon scripte, in quibus cartis sunt scripte septuagentas tregintas postem [...] Ego, idem, Petrus Bonfantini notarius predictus $\gg^{11}$. Questo farebbe pensare che il registro sia stato inviato alla camera actorum del comune, visto che normalmente i registri venivano descritti in questo modo negli inventari dell'archivio. Nell'inventario della camera actorum (1290-1303), sotto l' anno 1299 è registrato : " item recepi Petro domini Bonfantini Petriçoli, olim notario fratris Montis ad introytus unum librum in cartis pecudinis scriptum manu dicti Petri, continentem insse treginta quatuor cartas inter scriptas et non scriptas, quarum ex eis viginti octo et due dimidie sunt scripte et alie non scripte $\gg^{12}$. Grazie a questa indicazione possiamo affermare che il liber introituum del 1298 sia una scrittura definitiva destinata a essere conservata nell'archivio cittadino. A conferma di tutto ciò, c'è una riformagione aggiunta agli statuti cittadini, del 28 marzo 1295, la quale specifica che : «teneantur et debeant scribere introytus et expensas dicti massarii in cartis pecudinis et ipsos introitus et expensas exemplare per ordinem eorum propria manu in uno alio libro cartis pecudinis, scribendo quantitates per silabas, dictiones integras, et non per abriviaturam, ita quod sint duo libri eiusdem tenoris, unus quorum remaneat et remanere debeat penes dictos notarios depositarii, alter vero per dictos notarios ad cameram actorum deponatur infra unum mensem a die (exitu) eorum officii $\gg^{13}$. Gli altri libri introituum e i frammenti dei registri della tesoreria ancora conservati, riportano le indicazioni contabili costituite dai totali dei pagamenti registrati su ogni carta, nonché alla fine del mese e semestre ${ }^{14}$. Si tratta di dati che il tesoriere e i suoi notai utilizzavano per conoscere rapidamente le spese o le entrate dell'ufficio, che dovevano comunicare al consiglio cittadino e al giudice al sindacato, per la verifica del loro operato. La conferma di questa ipotesi è data dal fatto che un frammento di registro del 1303-1304, reca annotazione della verifica su di esso effettuata da parte di Vilani de Guastavillani, Iohannis de Ignatio, Romeo Pepolis e Ursi de Blanchitis, difensori dell'avere del comune (ufficio che sostituirà il giudice al sindacato nel controllo dell'amministrazione pubblica $)^{15}$.

10 A differenza dei libri introituum, i libri di spesa del 1288, del 1296 e del 1297 presentano caratteristiche compatibili con la funzione di registri preparatori. In essi i pagamenti sono singolarmente numerati in cifre romane, registrati in ordine cronologico (mese per mese) e corredati dalle somme delle spese di ogni pagina, di ogni mese e dell'intero registro. Oltre a questi espedienti funzionali a semplificare le operazioni di controllo, talvolta si aggiungono altri accorgimenti : ad esempio, nel caso del liber expensarum del 1297, a fianco del numero d'ordine che precede un pagamento a Benvenuto Gerardini, custode delle carceri, si avverte che i cinque compensi elargiti a quest'ultimo saranno per comodità contrassegnati tutti dalla medesima cifra (« reperiuntur isti quinque precepti in dicto signo $»)^{16}$. Quindi, grazie a questa nota è possibile stabilire che la numerazione romana corrisponde al precepto che ha autorizzato il pagamento, e quindi un elemento per semplificare l'operazione di controllo.

11 Per concludere, il registro del 1288 è dunque un registro di lavoro, scritto giorno per giorno da un notaio della tesoreria, e non un registro definitivo realizzato per essere inviato alla camera actorum del comune ${ }^{17}$.

\section{Struttura e caratteristiche}

L'incipit del registro spiega chiaramente il contenuto di tutto il documento e recita : « expensarum diversarum et variarum factarum per virum prudentem dominum Guillelmum de Pavanensibus civem et mercatorem civitatis Bononie depositarium generalem comunis Bononie tempore regiminis nobilis viri domini Bresani de Sali potestatis et capitanei civitatis 
Bononie sub millesimo ducentesimo octuagesimo octavo indictione prima et scriptum per me Paulum Bellondini notarium dicto officio deputatum $»^{18}$. Il registro è stato dunque scritto dal notaio Paulus Bellondini eletto alle dipendenze del depositario del comune, come previsto dagli statuti cittadini ${ }^{19}$. Nel registro sono raccolte in ordine cronologico tutte le spese del comune dal $1^{\circ}$ luglio al 31 dicembre 1288. A c.111r è presente un'aggiunta relativa a due pagamenti di giugno dell'anno seguente ${ }^{20}$. Il liber misura mm 340x $250^{21}$. In corrispondenza della carta $9 \mathrm{v}$ è possibile constatare la mancanza di alcune carte (che includono gli ultimi pagamenti del mese di luglio, e tutti i pagamenti dei primi quindici giorni del mese di agosto ${ }^{22}$. Una parte delle carte in questione sono state ritrovate in un altro fondo, quello dell' Ufficio dei Fortilizi e delle Munizioni dei Castelli $^{23}$. Non c'è alcun dubbio che si tratti del frammento del suddetto registro data la coincidenza di numerosi elementi ${ }^{24}$.

Il volume cartaceo è attualmente costituito da 111 carte, alle quali va aggiunto un quinione conservato nell' altro fondo indicato in precedenza, in buone condizioni di conservazione ${ }^{25}$. Il registro è privo di disposistivi di numerazione coevo delle carte, e inoltre bisogna rilevare che il supporto non presenta filigrane.

Il supporto ha poche macchie e qualche strappo nelle ultime quattro carte. L'inchiostro utilizzato è bruno e talvolta tendente al rossiccio. Il volume non si presenta come l'assemblaggio alluvionale di più quaterni. Il manoscritto non presenta spazi lasciati bianchi in vista di aggiunte successive. Il registro è scritto interamente in minuscola cancelleresca, opera di una sola mano, all'eccezione dei pagamenti relativi al giugno 1289 che presentano una scrittura di modulo maggiore.

Ogni carta presenta la stessa ripartizione funzionale dello spazio : uno specchio di scrittura provvisto di due margini (sul folio sono ben visibili le rigature a piombo). Sul margine sinistro sono indicate delle cifre romane in ordine crescente, corrispondenti a ogni pagamento registrato nella parte centrale della carta. La numerazione ricomincia ad ogni nuovo mese. Lo stesso sistema si riscontra anche in un liber expensarum del 1296, ma è assente nel liber expensarum del 1303-1304, e in tutti i registri successivi di spese conservati in archivio ${ }^{26}$. Si tratta quindi di una pratica utilizzata fino alla fine del XIII secolo e poi in seguito abbandonata. Probabilmente è il segno di un cambiamento nella gestione delle voci di spesa e nella revisione della documentazione contabile.

17 Quando la data è la stessa, il testo fa riferimento a eodem die o a espressioni simili rese in compendio. L'indicazione del mensile è scritta in maiuscolo in alto della parte centrale. Segno che testimonia l'intenzione di strutturare il registro in modo da facilitarne la consultazione, nell'intento di ritrovare rapidamente specifici pagamenti. Sono presenti poche cancellature e poche aggiunte interlineari, che sembrano appartenere alla stessa mano.

Il margine destro è utilizzato per indicare l'entità del pagamento, con i numeri scritti per esteso, o più raramente in cifre romane, con le abbreviazioni delle varie monete (lire, soldi e denari). La struttura del pagamento si ripete sempre allo stesso modo : dopo l'item solvit dictus depositarius o simile, sono esplicati il destinatario e il motivo della spesa, nonché il documento che la attesta e il notaio che l'ha rogato ${ }^{27}$. A volte, quando il pagamento ha come destinatari più persone, la parte centrale risulta suddivisa in due o tre colonne nelle quali sono inseriti i vari nomi sotto forma di lista, preceduti da un piede di mosca. Molte liste di destinatari hanno una rigatura verticale a piombo e parantesi per suddividere i nomi in sottogruppi.

In calce ad ogni pagina un'annotazione riassume le spese lì registrate (summa pagine). Lo stesso viene fatto alla fine di ogni mese e alla fine del semestre ${ }^{28}$. A volte nel margine sinistro, accanto alla cifra romana, si spiega la ragione del pagamento (per esempio, si annota castri Sancti Petri, per indicare un pagamento ai custodi di Castel San Pietro). Note utilizzate per velocizzare le operazioni di controllo del testo e del lavoro del tesoriere ${ }^{29}$.

\section{Note comparative}

20 Per la fine del XIII secolo si dispone di alcuni studi relativi ad altre città italiane, che presentano la fonte anche ne suoi aspetti codicologici e paleografici. Grazie ad rapido confronto del liber expensarum del 1288 con questa documentazione analoga e coeva di altre città sarà possibile 
rilevare le caratteristiche comuni e ipotizzare un'eventuale circolazione di modelli contabili. Ma ci sarebbe bisogno di uno studio più ampio per poter arrivare a delle conclusioni accettabili. Costituiscono un utile termine di confronto due documenti comunali di area veneta. Per la città di Verona si conserva un quaternus expensarum del novembre 1279, pergamenaceo, infolio, predisposto per la registrazione solo di alcune spese (stipendi di ambasciatori, giudici, ecc.) e verosimilmente destinato a essere rilegato con quaterni di altri mesi ${ }^{30}$. Per il comune di Treviso disponiamo di un quaternus del 1298, nel quale sono registrate le spese da luglio a settembre, disposte su tre fascicoli mm 420x300, con spazi bianchi, forse lasciati dai notai per successive aggiunte $^{31}$. Questa taglia lo allontana dal registro bolognese del 1288, ma è simile a quella di coevi frammenti di documentazione contabile dello stesso comune emiliano.

I casi veneti, mostrano dunque un tipo di registro diverso, assemblato mese per mese, mentre il liber expensarum di Bologna si presenta strutturato sin dal principio per poter accogliere un intero semestre di spese del comune. Sia quest'ultimi manoscritti, sia il registro bolognese, rispondono alle stesse necessità di contabilità e di controllo, secondo una suddivisione temporale delle spese, alla quale si potevano aggiungere eventuali ripartizioni tipologiche. All'Archivio Segreto Vaticano è conservato un registro di introiti e spese del 1279-1280 della corte di Niccolò III. Si tratta di un manoscritto cartaceo, mm 320x225, con registrazioni dei pagamenti in ordine cronologico e aggiunte relative al pontificato successivo ${ }^{32}$.

A differenza del liber bolognese del 1288, non sono presenti suddivisioni e nemmeno somme di uscite mensili e semestrali. Il Palmieri, che ha studiato questo registro, scrive : « questo libretto poi di dare ed avere, oltre l'importanza che ci offre dal lato filologico, ne ha anche un 'altra, per essere documento di civile podestà papale nella Romagna $»^{33}$, il che porta a ipotizzare che le caratteristiche simili siano frutto della circolazione di un medesimo savoirfaire amministrativo, ma che la contabilità sia tenuta in modo diverso, perché diverse sono le esigenze amministrative di un comune e di una corte papale.

\section{La spesa pubblica}

\section{Le ragioni della spesa}

Nel grafico appare la ripartizione dei settori di spesa.

\section{Fig. 1 - Le ragioni della spesa ${ }^{34}$}

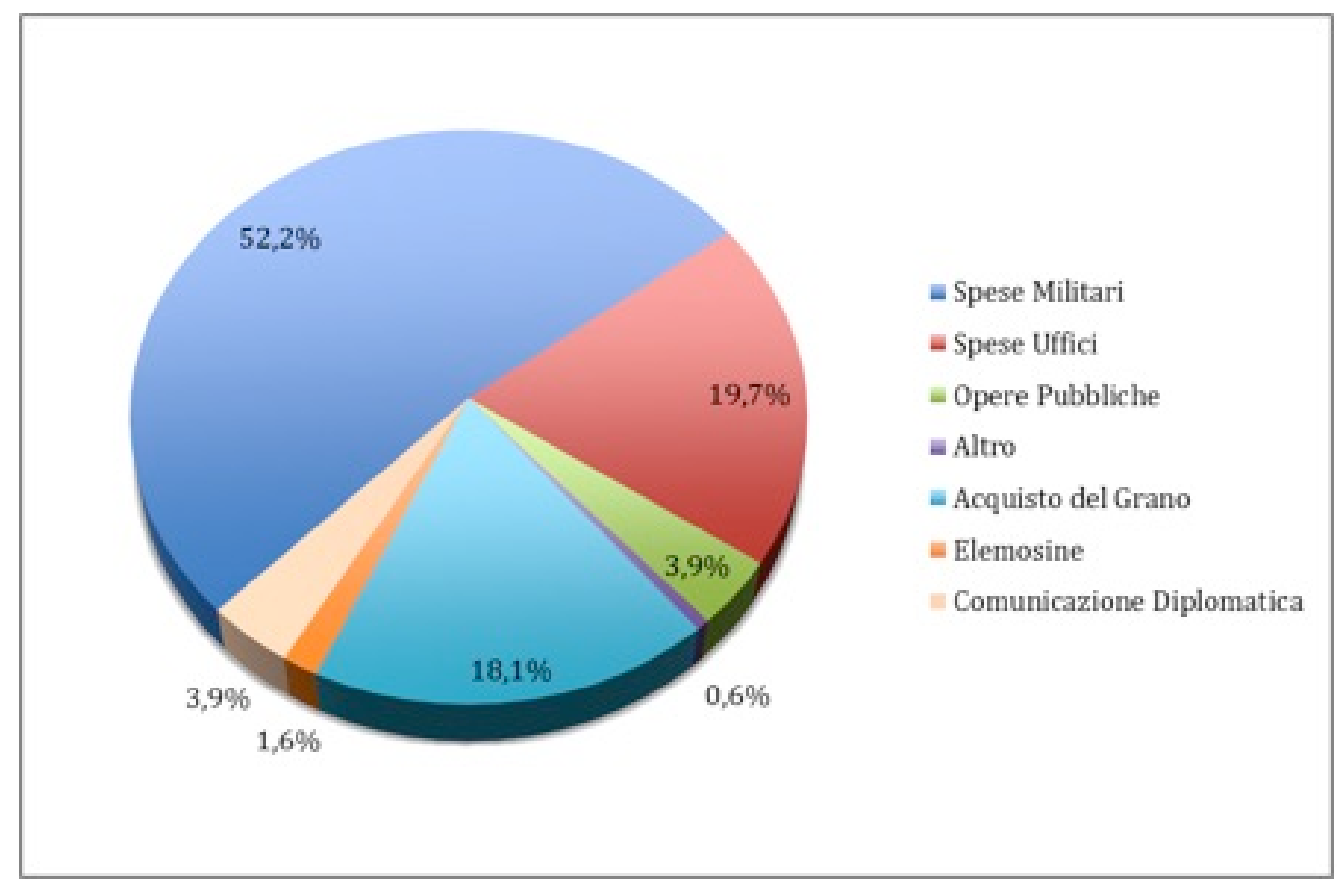

La spesa pubblica dei comuni italiani medievali può essere suddivisa in due tipi : quella ordinaria e quella straordinaria. Queste categorie erano perfettamente chiare al tempo della scrittura del registro. Nel libro undicesimo degli statuti della città del 1288 la terza rubrica si intitola : "De expensis ordinariis faciendis per comune Bononie ${ }^{35}$, e indica tutti gli ufficiali 
pubblici con il rispettivo salario, a partire dal podestà ( «pro feudo potestatis et ipsius familie, exceptis beroariis, duo milia centum libras bononinorum singulis sex mensibus $\gg)^{36} \mathrm{o}$ ancora « item omni anno octo trombatoribus comunis, silicet quatuor comuni et quatuor populi, quadragintas octuaginta libras bononinorum $\gg^{37}$. A queste spese ordinarie si riferiscono anche le elemosine che la città versava ad alcuni centri religiosi per delle festività.

Riguardo invece la parte straordinaria del bilancio, negli statuti possiamo trovare delle restrizioni relative alcuni pagamenti, ad esempio per il risarcimenti ai cavalieri. Il comune stabilisce nella rubrica «de extimatione equorum imponendorum per comune Bononie facienda. Rubrica. » che « estimati alicuius equi non ascendat ultra summam centum librarum bononinorum $»^{38}$.

Un dato importante da rilevare riguardo i bilanci mensili, è la disparità di spesa che può intercorrere tra un mese e l'altro. Ad esempio, la spesa d'agosto è all'incirca 1/12 di quella di dicembre. Questa disparità si inserisce in una tendenza generale in cui le spese straordinarie possono stravolgere completamente le dimensioni del bilancio. Il bilancio del mese di dicembre è quasi la metà del bilancio totale (che corrisponde a poco più di 41.500 lire) ${ }^{39}$ per effetto di un singolo pagamento, registrato il 20 dicembre, che concede 11.847 lire 4 soldi e 9 denari a 300 cavalieri, che prestarono servizio al comune per un anno ${ }^{40}$. Sempre nello stesso mese, si trovano altre due voci di grande entità : una di 2.048 lire $^{41}$ che corrisponde alla paga di alcuni cavalieri mercenari e l'altra di 2.000 lire per il depositarius generalis Guillelmum de Pavanensibus $^{42}$, per un prestito fatto al comune per il pagamento di alcuni mercenari ${ }^{43}$. Gli altri due mesi in cui si registrano uscite straordinarie rilevanti sono settembre e ottobre. In questo caso, pro solvendo datio bladi forensis ad civitatem Bononie, rispettivamente a settembre per 4.883 lire $^{44}$, e a ottobre per 2.500 lire $^{45}$. In ottobre vengono registrate anche altre spese notevoli : 1.194 lire per custodi e capitani da mandare a Reggio per il suo presidio ${ }^{46} ; 1.140$ lire e 8 soldi per il salario del podestà Corso Donati per i mesi di agosto, settembre, ottobre e parte del mese di luglio dell'anno in corso ${ }^{47}$. Per i restanti mesi non si segnala nessun pagamento di simili entità. La parte di bilancio straordinario occupa dunque i $3 / 5$ della spesa pubblica semestrale. Una delle ragioni determinati di questo dato è costituita dalle spese militari, corrispondenti al $52,2 \%$ del totale. Una parte di questa spesa era preventivabile dal comune, e precisamente quella parte relativa ai salari dei capitani e custodi dei castelli nel contado. Questi salari erano stabiliti anch'essi dalla rubrica sopradetta (anche se alcuni costituivano presidi straordinari, come Reggio o Monte Armato, quest'ultimo aggiunto solo in seguito negli statuti ${ }^{48}$. I pagamenti destinati a mantenere la sicurezza del contado erano i più numerosi. Ai centri del contado situati verso Firenze erano attribuiti meno custodi, dati i buoni rapporti con la città guelfa. Di converso, si stanziava un importo maggiore per la custodia delle località sul versante modenese e imolese-ravennate, che con Bologna avevano rapporti politici peggiori. I custodi erano guidati da un capitaneus, ed erano tutti eletti dal consiglio dei Duemila ad brevia ${ }^{49}$. Non sempre le paghe coincidono con quelle inserite nello statuto. Quest'ultimo indica 5 soldi al giorno per il capitano e 2 soldi e 6 denari per i custodi ${ }^{50}$, mentre di solito nel liber expensarum vengono registrati 4 soldi per il capitano e 2 soldi per il custode ${ }^{51}$. Lo scarto totale appare comunque abbastanza ridotto.

31 I cavalieri erano abitualmente assoldati per 15 giorni, per un massimo di due mesi ${ }^{52}$. Tra i pagamenti dei mercenari il più grande ammonta a 564 lire per 70 cavalieri inviati al servizio del Conte di Romagna ${ }^{53}$. I cavalieri mercenari sono pagati 8 o 12 lire al mese, come attestato in tutti i pagamenti a loro elargiti. La paga differisce poi in base al loro equipaggiamento, se costituito da uno o due cavalli. In un pagamento si fa distinzione se dotati di solamente un ronzino, o con ronzino e un equum armigerum ${ }^{54}$. I cavalieri muniti dei cavalli del comune costavano poco più di 3 lire al mese ${ }^{55}$, ma a differenza dei mercenari quest'ultimi potevano ottenere dei risarcimenti per eventuali danni alle loro cose e cavalli, e tutti di grande entità (per il secondo semestre del 1288 vengono registrati risarcimenti che si aggirano attorno le 20 lire ciascuno). Riguardo ai tempi dei risarcimenti dei cavalli è da notare che solo per due di loro è possibile stabilirlo, e cioè a novembre dell'anno precedente, quando fu in carica come podestà 
Gerardo de Iosano (1287). Per i primi due casi citati, tutti i pagamenti furono eseguiti dopo la prestazione del servizio. In totale il costo di tutti i risarcimenti ammonta a 139 lire e 10 soldi $^{56}$. La fanteria, anche se meno cara, costituisce comunque una parte importante della spesa : l'invio di 100 fanti a Reggio per un mese costa 456 lire. Il sistema di arruolamento era quello delle venticinquine, 25 uomini per quartiere con un capitano alla loro guida, il capitano era pagato 4 soldi al giorno, mentre i custodi 3 soldi giornalieri ${ }^{57}$. Sempre in ambito militare sono presenti altre spese di piccola entità come l'acquisto di seta per 3 lire, per farne un pennone con le insegne di Carlo Martello d'Angiò per la custodia di Reggio ${ }^{58}$.

L'altra grande voce di spesa straordinaria era quella destinata all'acquisto del grano, corrispondente nel nostro registro al $18,1 \%$ del totale. A tal proposito sono registrati solo tre pagamenti : quello di settembre è di 4.883 lire, quello di ottobre 2.500 lire, quello di novembre di 117 lire. La politica annonaria di Bologna era a differenza di altre città abbastanza rigida, favoriva solo l'importazione e limitava le speculazioni ${ }^{59}$. Tra le spese meno onerose, si possono annoverare la diplomazia, le opere pubbliche e poco altro. Il 3,9\% del bilancio era speso per quella che potremmo chiamare la comunicazione diplomatica, cioè per pagare i funzionari inviati ai confini o fuori dal contado per negoziare trattati, informare ed informarsi della situazione politica. Tutte queste operazioni erano ben remunerate. Quattro ambasciatori sono pagati 14 lire e 8 soldi per recarsi dai signori di Ferrara per quattro giorni ${ }^{60}$, mentre risultano pagate 4 lire e 10 soldi ad Antholino domini Rolandini de Thebaldis inviato a Modena per alcuni secretis negotiis ${ }^{61}$. Una decina di volte gli ambasciatori si recano a Roma, un po' meno verso le città guelfe fuori la Romagna, come Arezzo e Firenze, per informarsi sui conflitti in corso e trattare questioni della parte. Le più numerose sono le ambasciate verso il Conte di Romagna e gran parte della città della regione, Parma, Modena, Imola, Ravenna, Reggio, Cesena, Ferrara, o verso membri della famiglia Estense (interessante un pagamento per la scrittura di atti di concordia con il legato papale Bertoldo Orsini) ${ }^{62}$. Oltre agli ambasciatori risultano delle spese anche per delle spie : il 9 agosto ne furono inviate due, una nella zona di Mantova e l'altra nei dintorni di Pisa, la prima pagata 21 soldi, la seconda 25 soldi $^{63}$.

Un'uguale percentuale del 3,9\% del bilancio risulta destinata alle opere pubbliche : piccoli lavori all'interno di palazzi e uffici del comune, riparazioni o costruzioni di edifici nel contado. Per i lavori nel contado, i pagamenti più rilevanti ammontano a 850 lire (per alcuni lavori a Bargi), e a 200 lire (per due riparazioni di mura, torri, e ponti in alcune località del contado) ${ }^{64}$. Possiamo riscontrare anche spese di ordine minore come la riparazione di due porte del borgo di San Marino, che costò 6 soldi $^{65}$. In città, i lavori più cari furono eseguiti per il naviglio (50 lire), e per alcune riparazioni nel palazzo del comune ( 25 lire $)^{66}$. Dopo altre spese, di un ordine di grandezza di poco inferiore, si trovano lavori di piccola entità per la riparazione di serrature, chiavi, finestre, e altro ${ }^{67}$. Il registro consente di ricavare anche informazioni su alcuni prezzi, ad esempio, una corba di gesso costava 15 denari, l'istallazione di una serratura dai 3 ai 4 soldi, e una chiave da 12 a 18 denari $^{68}$.

Infine risulta un pagamento per una pittura infamante. La pittura fu eseguita da Zechonia (Cicogna di Rolando), artista molto attivo in quegli anni, e Corvo ${ }^{69}$, ai danni di Rambertus de Baçaleriis $^{70}$, rispettivamente nel palazzo vecchio e nuovo del comune. È interessante notare che Ramberto era stato inserito nella rubrica « de satisdatione prestanda ab infrascriptis nobilibus civitatis vel districtus Bononie, et de penis eorum qui non darent dictam securitatem et de fidantia eis danda, ratione predicta $\gg^{71}$. Si trattava quindi di un magnate. È quindi presumibile che dopo esser stato bandito fu dipinto a monito di tutti gli altri magnati ${ }^{72}$. Infine, nella categoria che abbiamo definito "altro", si trovano ufficiali non catalogabili nei gruppi sopradetti che offrono servizi vari : prestazioni di medici, cattura di banditi (ricompensata con 50 lire), ecc. ${ }^{73}$. La somma pagata per questo servizio è piuttosto ragguardevole, e mostra il grande investimento economico del comune nei riguardi dell'ordine pubblico. In questa categoria vanno inseriti anche i prestatori di servizi particolari, come i due marochi, Iohannino de Brisia e Baxanino de Mediolano, che andarono con un notaio cittadino ad capiendum porchos ${ }^{74}$. Da notare infine, l'acquisto di un ronzino, un falco e di altre cose per 
una prova equorum nel sesto di San Bartolomeo per la somma di 19 lire e 14 soldi, e 12 denari spesi per l'acquisto di due capistris (museruole) per dei leoni del comune, simbolo della città di Bologna ${ }^{75}$.

Una volta messa da parte tutte queste spese straordinarie, le voci di spesa preventivabile restano di fatto due : quella per gli uffici pubblici e quella per le elemosine, che ammontano rispettivamente al $19,7 \%$ e al 1,6\% delle uscite del registro.

La spesa destinata agli uffici comprendeva principalmente tutti i salari degli ufficiali pubblici, ma anche compensi per servizi specifici, l'acquisto di strumenti di lavoro, e tutto quello che serviva al loro funzionamento. I pagamenti più ingenti appaiono quelli destinati ai funzionari forestieri, i cui salari corrispondono precisamente a quelli previsti negli statuti. Normalmente sono pagati mese per mese, a parte il caso del podestà Corso Donati, che fu pagato in ritardo di più di 3 mesi, così come i suoi berrovieri ${ }^{76}$. La categoria più presente e trasversale è ovviamente quella dei notai, i quali si occupavano di tutta la scrittura di atti e registri essenziali per il funzionamento di ogni ufficio pubblico. Così si pagano dei notai «pro confectione et completura licterarum missarum domino Guillelmo de Oddonis secondo ellecto in capitaneis populi » o un notaio con altri ufficiali « ituri ad denuntiandum domino Bindo Bascherie ellecto primo in capitaneis populi $\gg$ o ancora per la scrittura dei brevi per il consiglio dei Duemila $^{77}$. Sempre i notai, insieme ad altri, erano coloro che si occupavano delle stime delle proprietà della parte dei Lambertazzi, o « ad inquirendum confinatos comunis Bononie pro parte Lambertaciorum », tutte operazioni che costavano parecchio al comune. La prima azione costò 75 lire mentre la seconda 18 lire e 15 soldi $^{78}$. Queste cifre danno un idea della spesa derivante dalla politica antighibellina, e della speranza che il comune riponeva negli affitti di questi beni per ricavarne il più possibile denaro. In questo senso vanno lette le provvigioni per aumentare gli scarsi redditi ricavati da questi beni ${ }^{79}$. I vari notai che prestano servizio al comune presso gli uffici sono pagati nel seguente modo : 4 lire per i notai ad malleficia (cioè coloro che lavoravano nei tribunali criminali), 3 lire per tutti gli altri. Dal registro risulta inoltre che per sei mesi furono pagati 6 notai al servizio del disco dell'orso, mentre nello statuto si destinano a questo ufficio solamente 4 notai. Nel pagamento riferito al loro stipendio sono anche inseriti due notai ad discum vulpis (ufficio non indicato negli statuti e non conteggiato nel calcolo del pagamento ${ }^{80}$.

Altri salari coincidono perfettamente con quelli previsti dallo statuto : 240 lire per gli approbatori, o ancora 12 lire al notarium ad cassam per sei mesi, anche se pagato mese per mese $^{81}$. Infine si trovano ufficiali pagati per compiti specifici ed occasionali : ad ottobre sono pagati coloro che scrissero e parteciparono alla stesura degli statuti del 1288 , o ancora notai che hanno accompagnato ambasciatori, messaggeri che hanno assistito ad esecuzioni, e altro ancora $^{82}$.

41 Numerosi sono anche i pagamenti destinati ai banditori e ai messaggeri del comune che venivano inviati nel contado ad cytandum, ad cridandum, o ad requirendum ${ }^{83}$. Il loro costo era di 2 soldi per ogni giorno di viaggio, ma a causa dei frequenti servizi e di qualche salario più alto si superano le 300 lire di spesa. Legittimo pensare che questa spesa legata alla giustizia andò ad aumentare soprattutto in questi anni visto che tra il 1286 e il 1296 si assiste ad un aumento costante dei processi penali accusatori, che finiscono per essere 650-700 per semestre, conseguenza dell'apogeo del popolo a scapito del potere del podestà e del capitano del popolo ${ }^{84}$. Altri costi relativi all' amministrazione della giustizia risultano essere le esecuzioni o la fustigazione di prigionieri, circostanze nelle quali sono chiamati ancora dei messaggeri come testimoni dell'esecuzione, pagati 12 denari per questo servizio ${ }^{85}$. Infine, si registrano tutte le spese relative l'acquisto di carta, pane destinato ai carcerati, olio per le lampade ed altro ${ }^{86}$. Per di più, bisogna evidenziare che la carta era normalmente acquistata dagli ufficiali comunali per i loro uffici, ma non è mai indicata la quantità. Invece possiamo conoscere il prezzo della cera che è di 4 soldi ogni libbra ${ }^{87}$.

In conclusione, il restante $1,6 \%$ dei pagamenti è destinato ai religiosi di conventi e chiese, per lo più per elemosine in occasioni di festività religiose, per l'acquisto di ceri o per lavori 
agli edifici che li ospitano. I pagamenti più consistenti destinati alle elemosine sono compiuti in moneta minuta, a differenza di tutti gli altri. Queste elemosine sono regolate dagli statuti ${ }^{88}$. Per la chiesa di San Giovanni in Monte e Sant'Ambrogio le cifre corrispondono (10 lire), mentre per la festività di Santo Stefano, anch'essa fissata a 10 lire, il pagamento nel registro è superiore, e ammonta a 25 lire $^{89}$.

\section{La spesa pubblica e gli ufficiali comunali}

Nel grafico seguente sono indicati i destinatari della spesa suddivisi tra : ufficiali del comune (notai, ambasciatori, messaggeri, ecc.) ; ufficiali forestieri (capitano del popolo, podestà, berrovieri, ecc.) ; prestatori di servizi (muratori, fabbri, falegnami, pittori, medici, ecc.) ; soldati (fanti, cavalieri, mercenari, custodi dei castelli, ecc.) ; comunità religiose.

\section{Fig. 2 - Distribuzione della spesa per numero di persone}


= Prestatori di Servizi

= Ufficiali Comunali

Osservando il grafico n.2 è subito evidente come il $60 \%$ delle persone che in quel semestre furono pagate dal comune di Bologna avevano servito come fanti, cavalieri, custodi dei castelli e mercenari. Quest'ultimi, come visto pesavano fortemente sul bilancio comunale ${ }^{90}$. I custodi dei castelli venivano eletti ad brevia, invece i cittadini sarebbero stati arruolati secondo il sistema delle venticinquine ${ }^{91}$. In totale si trattava di più di un migliaio di persone pagate in un semestre, e tra di loro poco più di $1 / 4$ erano forestieri, gli altri tutti soldati reclutati in città. Un gruppo inferiore della metà (29\% dei pagati) è quello degli ufficiali della città ai quali si aggiunge il piccolo gruppo degli ufficiali forestieri che corrispondono al $2 \%$ del totale. Pittori, muratori, falegnami, fabbri, ecc., corrispondono al $8 \%$ del totale (all'incirca 160 persone), mentre il restante $1 \%$ appartiene al gruppo dei religiosi a capo di conventi e chiese.

L'aspetto più interessante è quello delle persone che ricoprivano delle cariche elettive. Dalla lettura degli statuti sembrerebbe che ogni anno circa 1800 persone erano occupate a vario titolo come ufficiali comunali. Il registro tuttavia mostra che numerose cariche potevano essere prorogate oltre la scadenza. Nel registro per esempio ci sono casi di proroghe, da 3 a 39 giorni, per il mandato ai capitani e custodi (in teoria avevano un mandato mensile) ${ }^{92}$. Di conseguenza, in realtà, gli incarichi non erano 12 all' anno ma in media tra 8 e 10 . Lo stesso registro mostra poi che era possibile ricoprire la stessa carica, anche più volte l'anno. Dunque ogni anno le persone pagate come ufficiali del comune non erano 1800 , cioè tanti quanti gli incarichi previsti dagli statuti, ma un numero inferiore, oscillante tra poco meno di 1200 e 1500 persone $^{93}$. Se consideriamo questo dato congiuntamente alla totalità delle spese del registro ricaviamo che nel corso di un semestre più della metà delle persone effettivamente pagate dal comune ricoprivano una carica elettiva e di conseguenza che circa la metà del bilancio delle spese era destinata a cittadini eletti.

48 Antonio Ivan Pini ha dimostrato che a Bologna tra il 1273 e il 1306, le cosiddette persone "atte alle armi", cioè gli uomini tra 18 e 70 anni, erano poco più di 5.200/5.30094. Ciò implica 
che più di 1/4 degli "atti alle armi" della popolazione bolognese erano eletti dal consiglio dei Duemila per ricoprire degli incarichi comunali ogni anno.

Questo consiglio era dunque un importantissimo centro di smistamento degli incarichi e dunque dei salari ad essi connessi, veniva a rappresentare uno dei canali fondamentali per la distribuzione delle risorse pubbliche ${ }^{95}$.

Va osservato tuttavia che, a parte alcune cariche particolarmente ben pagate come quelle dei giudici, dei notai ai dischi, dei cancellieri del comune o del notaio degli anziani e dei consoli (quest'ultimi pagati 6 lire al mese ${ }^{96}$ ), le paghe degli altri ufficiali non erano molto elevate. Ad esempio : un notaio ad cassam comuni era pagato 40 soldi per un mese di carica ${ }^{97}$; il salario dei custos massarie comunis era di 12 lire per sei mesi ${ }^{98}$; e i messaggeri del comune erano pagati due soldi al giorno ${ }^{99}$. Visto che i muratori venivano pagati 3-4 soldi al giorno ${ }^{100}$, un pittore per un lavoro guadagnava dalle 20 alle 40 lire $^{101} \mathrm{e}$ un medico per alcune cure ed un amputazione era pagato 10 soldi $^{102}$, sembrerebbe che le cariche elettive, a parte qualche eccezione, non fossero poi così appetibili. D'altra parte chi era eletto non doveva necessariamente abbandonare il proprio lavoro e poteva accumulare più cariche nello stesso anno.

\section{Conclusioni}

51 Proviamo ora a tirare le fila del discorso, per comprendere quali fossero i principali aspetti della spesa pubblica a Bologna nel 1288 così come emergono dall' analisi del liber expensarum.

In primo luogo appare notevole la sproporzione tra parte preventivabile e parte straordinaria del bilancio. Quest'ultima ammonta a più del doppio del totale ed è determinata in primo luogo dall'aumento delle spese militari, pari al 52,2 \% del semestre. Nel 1287 le spese per la guerra erano sempre più ingenti, a causa dell'aggressione da parte delle potenze vicine sul fronte occidentale ed orientale. Nel 1288 le minacce degli Estensi si erano ormai fatte concrete, le lotte tra i ghibellini e guelfi si erano moltiplicate e i legati papali non erano riusciti a mantenere una pace duratura. Alla fine degli anni ottanta, Bologna indeboliva le sue finanze anche per l'invio di truppe al Conte di Romagna, come registrato in numerose occasioni. Questi conflitti avevano già assorbito numerose risorse quando, nel secondo semestre del 1288, si aprì un contenzioso anche con Reggio. Inoltre, Bologna spendeva molto per la difesa del suo contado, che già da metà Duecento contava una trentina d'insediamenti a nord della via Emilia, e un'altra trentina a sud. La risposta del comune al movimento di accerchiamento messo in atto dai signori di Ferrara fu il rafforzamento delle posizioni già acquisite in Romagna, tramite ristrutturazioni e nuove fortificazioni che pesarono molto sulla spesa pubblica. La scelta di Bologna di rimanere nell'orbita guelfo-angioina gravava quindi notevolmente sulle casse della città, anche a causa dell'istituzione di provvedimenti speciali, come il premio di 25 lire a chi desse in potere del comune un bandito ghibellino, o di tutti i costi della gestione dei beni della parte dei Lambertazzi ${ }^{103}$.

Altro notevole costo era quello destinato al funzionamento degli uffici del comune, che equivaleva per spesa al $19,7 \%$ del totale. La macchina comunale funzionava grazie a 1300/1500 cittadini eletti ogni anno ai vari uffici che coinvolgevano dunque più di $1 / 4$ della popolazione maschile in condizione di parteciparvi. Benché le spese preventivate dagli statuti comunali per i salari degli ufficiali fossero in sostanza rispettate, ad esse si aggiungevano uscite di altro genere, che andavano ad ampliare ulteriormente il dislivello tra le due parti del bilancio.

Infine, a queste due voci bisogna aggiungere quella dell'acquisto del grano, che pesava per il $18,1 \%$. Questo costo può essere considerato in una certa misura straordinario, dato che poteva essere notevolmente aumentato da una carestia, o anche da un' annata meno produttiva ${ }^{104}$. Ma anch'essa era resa più ingente dalla guerra, come sembra dimostrare il fatto che, a giudicare dai termini di confronto forniti da Hessel, le spese del secondo semestre del 1288, appaiono notevolmente aumentate rispetto alla metà dello stesso secolo, ma non differiscono poi molto da quelle di un semestre del 1271 quando Bologna era in guerra su altri fronti ${ }^{105}$. Un ultimo dato che emerge è la parziale incapacità della città di pagare in tempi ridotti, ulteriore segnale del precario equilibrio tra entrate, spese e debito pubblico. 
Possiamo ipotizzare che le spese relative agli ufficiali rimasero dello stesso ordine di grandezza anche per gli anni seguenti, dal momento che non vi furono grandi rinnovamenti della struttura amministrativa del comune. Riguardo ai conflitti, sarebbero in parte cambiati i fronti ma le forze messe in gioco dal comune non sarebbero state molto superiori a quelle mobilitate nel 1288. Infine per la voce che si riferisce al grano, dato che la popolazione non subì variazioni numeriche notevoli, possiamo supporre che le necessità di approvvigionamento della città si mantennero sugli stessi livelli.

Risulta in ogni caso confermato che furono le spese straordinarie, e soprattutto militari, e in misura minore i pagamenti relativi all'acquisto di grano a creare forti differenze tra i bilanci delle varie mensilità. Questi due elementi resteranno nei bilanci comunali e signorili come le spese più importanti, che richiederanno i maggiori sforzi per non aumentare esponenzialmente i debiti pubblici cittadini. Bologna risulta dunque allineata alla maggior parte delle realtà comunali italiane, che vedono le loro uscite aumentare notevolmente a partire all'incirca dall'ultimo quarto del tredicesimo secolo a causa dell'intensificarsi della competizione politica $^{106}$.

\section{Bibliographie}

Acta Comunitatis Tarvisii 1998 = A. Michelin, G. M. Varanini (a cura di), Gli Acta Comunitatis Tarvisii del sec. XIII, Roma, 1998, p. 899-987.

Albini 1998 = G. Albini, Le scritture del comune : amministrazione e memoria delle città dei secoli XII e XIII, Torino, 1998.

Approche codicologique $2011=$ Approche codicologique des documents comptables du Moyen Âge, in Comptabilités, 2, 2011 (disponibile in linea all'indirizzo : http://comptabilites.revues.org/368 (ultima consultazione in data 29/08/2016)).

Baietto $2000=$ L. Baietto, Scrittura e politica. Il sistema documentario dei comuni piemontesi nella prima metà del secolo XIII, in Bollettino storico-bibliografico subalpino, 98, 2000, p. 105-165, 473-528.

Baietto 2002 = L. Baietto, Elaborazione di sistemi documentari e trasformazioni politiche nei comuni piemontesi (secolo XIII) : una relazione di circolarità, in Società e storia, 98, 2002, p. 645-679.

Bartoli Langeli 2006 = A. Bartoli Langeli, Notai. Scrivere documenti nell'Italia medievale, Roma, 2006.

Blanshei $2010=$ S. R. Blanshei, Politics and justice in late medieval Bologna, Boston, 2010.

Bocchi 1973 = F. Bocchi, Le imposte dirette a Bologna nei secoli XII-XIII, in Nuova rivista storica, 57 , 1973, p. 273-312.

Bocchi 1981 = F. Bocchi, Imposte dirette e ceti sociali a Bologna in età comunale, in Cultura e scuola, 77, 1981, p. 99-106.

Bocchi 1987 = F. Bocchi, L'azienda Santo Stefano, in F. Bocchi (a cura di), Sette colonne e Sette Chiese, la vicenda ultramillenaria del complesso di S. Stefano in Bologna, Bologna, 1987, p. 183-210.

Bordone 1984 = R. Bordone, La società urbana nell'Italia comunale (secoli XI-XIV), Torino, 1984. (disponibile in linea all'indirizzo : http://www.rm.unina.it/didattica/fonti/bordone/indice.htm (ultima consultazione in data 29/08/2016)).

Bowsky 1976 = W. M. Bowsky, Le finanze del comune di Siena (1287-1355), Firenze, 1976.

Castagnini 1976 = O. Castagnini, Il patrimonio di un frate gaudente bolognese all'inizio del Trecento : Dondiego Piantavigne, in Il Carrobbio, 2, 1976, p. 103-125.

Chittolini 2002 = G. Chittolini, "Fiscalité d'État » et prérogatives urbaines dans le Duché de Milan à la fin du Moyen Âge, in P. Contamine, J. Kerherve, A. Rigaudière (a cura di), L'impôt au Moyen Age : l'impôt public et le prélèvement seigneurial, fin XIIe-début XVIe siècle, I (Le droit d'imposer) (Bercy, 14-16 juin 2000), Parigi, 2002, p. 147-176.

Cittadini in armi 2011 = P. Grillo (a cura di), Cittadini in armi : eserciti e guerre nell'Italia comunale, Catanzaro, 2011.

Comptabilités pontificales 2006 = A. Étienne, T. Valérie (a cura di), Les comptabilités pontificales, in Mélanges de l'École française de Rome-Moyen Âge, 118/2, 2006, p. 165-268.

Cova $2010=$ P. Cova, Nuovi studi sulla miniatura delle matricole e degli statuti delle confraternite medievali bolognesi, in Rivista di storia della miniatura, 14, 2010, p. 81-97. 
Democrazia diretta 1997 = G. Schiavone (a cura di), La democrazia diretta. Un progetto politico per la società di giustizia, Bari, 1997.

Disette dans la conjoncture 2011 = M. Bourin, J. Drendel e F. Menant (a cura di), Les disettes dans la conjoncture de 1300 en Méditerranée occidentale, Roma, 2011.

Filippini - Zucchini 1947 = F. Filippini, G. Zucchini, Miniatori e pittori a Bologna. Documenti dei secoli XIII e XIV, Firenze, 1947.

Frescura Nepoti 1982 = S. Frescura Nepoti, Natura ed evoluzione dei dazi bolognesi nel secolo XIII, in Atti e memorie della Deputazione di Storia Patria per le Province di Romagna, 31-32, 1982, p. 136-166.

Foschi 1989 = P. Foschi, Il castello imperiale di Bologna : ricerche topografiche e urbanistiche, in J. Ortalli, C. De Angelis e P. Foschi (a cura di), La rocca imperiale di Bologna, Bologna, 1989, p. 71-98.

Giansante 1985 = M. Giansante, Il quartiere bolognese di Porta Procola alla fine del Duecento. Aspetti economici e sociali dell'estimo del 1296-7, in Il Carrobbio, 11, 1985, p. 123-141.

Giansante 1985-1986 = M. Giansante, L'età comunale a Bologna. Strutture sociali, vita economica e temi urbanistico-demografici : orientamenti e problemi, in Bullettino dell'istituto storico italiano per il Medio Evo e Archivio muratoriano, 92, 1985-86, p. 102-222.

Ginatempo 2000 = M. Ginatempo, Prima del debito. Finanziamento della spesa pubblica e gestione del deficit nelle grandi città toscane (1200-1350), Firenze, 2000.

Ginatempo 2013 = M. Ginatempo, Il finanziamento del deficit pubblico nelle città dell'Italia centrosettentrionale, in G. De Luca, a. Moioli (a cura di), Debito pubblico e mercati finanziari in Italia. Secolo XIII-XX, Milano, 2007, p. 39-82 (disponibile in linea all'indirizzo : http://www.dssbc.unisi.it/sites/ st03/files/allegatiparagrafo/21-052013/ginatempo_finanziamento1.pdf (ultima consultazione in data 29/08/2016)).

Greci - Pini 1976 = R. Greci, A. I. Pini, Una fonte per la demografia storica medievale : "Le Venticinquine” Bolognesi (1247-1404), in Rassegna degli Archivi di Stato, 36, 1976, p. 337-417.

Grillo 2001 = P. Grillo, Milano in età comunale (1183-1276). Istituzioni, società, economia, Spoleto, 2001.

Grillo 2006 = P. Grillo, «Reperitur in libro ». Scritture su registro e politica a Milano alla fine del Duecento, in G.G. Merlo, C. Richelmi (a cura di), Libri, e altro. Nel passato e nel presente, Milano, 2006, p. 32-53.

Grillo 2007 = P. Grillo, « 12.000 uomini, di cui 6000 con lance lunghe e 3.000 con pancere e mannaie ». L'esercito milanese all'inizio del Trecento, in Società e Storia, 116, 2007, p. 233-253.

Grillo 2008 = P. Grillo, Cavalieri e popoli in armi : le istituzioni militari dell'Italia medievale, RomaBari, 2008.

Hessel 1975 = A. Hessel, Storia della città di Bologna. 1116-1280, Bologna, 1975. (1a ed. Berlino 1910).

Knapton 1997 = M. Knapton, La dinamica delle finanze pubbliche, in G. Arnaldi, G. Cracco, A. Tenenti (a cura di), Storia di Venezia. dalle origini alla caduta 3, Roma, 1997, p. 475-528.

Knapton 1998 = M. Knapton, Il controllo contabile nello « Stato da Terra » dellla Repubblica Veneta : Norme comportamenti e problemi a Padova verso fine 400', in S. Zambon (a cura di), Metamorfosi dello stato e controllo contabile nella Repubblica veneta : elementi introduttivi, Bologna, 1998, p. 107-148.

Lazzari 2011 = T. Lazzari, Milites a Imola : la lista dei cavalli (1319) e la struttura sociale urbana, in M. Bassetti, A. Ciaralli, M. Montanari, G. M. Varanini (a cura di), Studi sul Medioevo per Andrea Castagnetti, Bologna, 2011, p. 219-240.

Lazzarini 2001 = I. Lazzarini, Transformations documentaires et analyses narratives au XVe siècle : les principautés de la plaine du Pô sub specie scripturarum, in Mélanges de l'École française de RomeMoyen Âge, 113, 2001, p. 699-721.

Lazzarini 2003 = I. Lazzarini, L'Italia degli Stati territoriali : secoli XIII-XV, Roma, 2003.

Livi 1933 = G. Livi, Autografi di Pier de' Crescenzi nel Reale Archivio di Stato di Bologna, in Pier de' Crescenzi (1233-1321). Studi e documenti, Bologna, 1933, p. 61-76.

Magni 2015 = S. G. Magni, Politica degli approvvigionamenti e controllo del commercio dei cereali nell'Italia dei comuni nel XIII e XIV secolo : alcune questioni preliminari, in MEFRM, 127/1, 2015 URL : http://mefrm.revues.org/2473.

Mainoni 2007 = P. Mainoni, Cremona Ytalie quondam potentissima. Economia e finanza pubblica nei secoli XIII-XIV, in G. Andenna e G. Chittolini (a cura di), Storia di Cremona. Il Trecento. Chiesa e cultura (VIII-XIV secolo), Cremona, 2007, p. 318-373. 
Maire Vigueur 2004 = J.C. Maire Vigueur. Cavalieri e cittadini $:$ guerra, conflitti e società nell'Italia comunale, Bologna, 2004. (1a ed. Parigi 2003).

Micheletti 1981 = D. Micheletti, Gli estimi del comune di Bologna il quartiere di Porta Ravennate (1296-97), in Il Carrobbio, 7, 1981, p. 293-304.

Milani 2003 = G. Milani, L'esclusione dal comune, conflitti e bandi politici a Bologna e in altre città italiane tra XII e XIV secolo, Roma, 2003.

Milani 2010 = G. Milani, Il peso della politica sulla mobilità sociale (Italia comunale 1300), in S. Carocci (a cura di), La mobilità sociale nel Medioevo. Atti del IV Incontro su Économies et Sociétés Médiévales. La Conjoncture de 1300 en Méditerranée Occidentale, (Roma 28-31 maggio 2008), Roma, 2010, p. 409-436.

Milani 2012 = G. Milani, Bologna, Spoleto, 2012.

Milani 2015 = G. Milani, Appunti sul finanziamento del disavanzo a Bologna in età comunale (1250-1274), in L. Capo, A. Ciaralli (a cura di), Per Enzo. Studi in memoria di Vincenzo Matera, Firenze, 2015, p. 179-209.

Orlandelli 1951 = G. Orlandelli, La revisione del bilancio nel Comune di Bologna dal XII al XV secolo, in Atti e Memorie della Deputazione di Storia Patria per le Province di Romagna, 2, 1951, p. 157-218.

Orlandelli 1954 = G. Orlandelli, Gli uffici economici e finanziari del comune dal XII al XV secolo. Inventario dell' Archivio di Stato di Bologna, Roma, 1954.

Ortalli $1979=$ G. Ortalli, Pingatur in palatio. La pittura infamante nei secoli XIII-XVI, Roma, 1979.

Palmieri 1889 = G.N. Palmieri, Introiti ed esiti di Papa Niccolò III, 1279-1280, Roma, 1889.

Pini 1977 = A. I. Pini, Gli estimi cittadini di Bologna dal 1296 al 1329, un esempio di utilizzazione : il patrimonio fondiario del beccaio Giacomo Casella, in Studi Medievali, 18, 1977, p. 111-159.

Pini 1996 = A.I. Pini, Città medievali e demografia storica : Bologna, Romagna, Italia : Secc. XIII-XV, Bologna, 1996.

Pinto 1978 = G. Pinto, Il libro del Biadaiolo. Carestie e annona a Firenze dalla metà del 200' al 1348, Firenze, 1978.

Politiche finanziarie e fiscali 2001 = P. Mainoni (a cura di), Politiche finanziarie e fiscali nell'Italia settentrionale (secoli XIII-XV), Milano, 2001.

Romiti 1994 = A. Romiti, L'Armarium Comunis della Camera actorum di Bologna. L'inventariazione archivistica nel XIII secolo, Roma, 1994.

Sella 1933 = P. Sella, Decima di Bologna dell'anno 1300, in E. Nasalli Rocca, P. Sella (a cura di), Rationes decimarum italiae nei secoli XIII e XIV. Aemilia : le decime dei secoli XIII-XIV, Città del Vaticano, 1933, p. 225-270.

Smurra $2003=\mathrm{R}$. Smurra, Prassi amministrativa e spazi di circolazione come immagine urbana : Bologna alla fine del Duecento, in F. Bocchi, R. Smurra (a cura di), Imago Urbis. L'immagine della città nella storia d'Italia. Atti del Congresso Internazionale (Bologna, 5-7 settembre 2001), Bologna, 2003, p. 417-434.

Smurra 2007 = R. Smurra, Città, cittadini e imposta diretta a Bologna alla fine del Duecento. Ricerche preliminari, Bologna, 2007.

Società dei notai 1988 = G. Tamba (a cura di), La società dei notai di Bologna, Roma, 1988.

Statuti del comune 1869-1884 = L. Frati (a cura di), Statuti del comune di Bologna dell'anno 1245 all'anno 1267, Bologna, 1869-1884.

Statuti di Bologna 1939 = G. Fasoli e P. Sella (a cura di), Statuti di Bologna dell'anno 1288, Città del Vaticano, 1939.

Storia di Bologna 2007 = O. Capitani (a cura di), Storia di Bologna, II. Bologna nel Medioevo, Bologna, 2007.

Tamba 1982 = G. Tamba, Consigli elettorali degli ufficiali del Comune bolognese, in Rassegna degli Archivi di Stato, 42, 1982, p. 34-95.

Trombetti Budriesi 2014 = A. L. Trombetti Budriesi, Gli statuti di Bologna e la normativa statutaria dell'Emilia Romagna tra XII e XVI secolo, in Mélanges de l'École française de Rome-Moyen Âge, 126/2, 2014, URL : https://mefrm.revues.org/2396.

Vallerani 2005 = M. Vallerani, La giustizia pubblica medievale, Bologna, 2005. 
Vallerani 2009 = M. Vallerani, « Ursus in hoc disco te coget solvere fisco ». Evasione fiscale, giustizia e cittadinanza a Bologna fra Due e Trecento, in Credito e cittadinanza nell'Europa mediterranea dal medioevo all'età moderna, (Asti, 8-10 ottobre 2009), p. 39-50

Vallerani 2013 = M. Vallerani, Logica della documentazione e logica dell'istituzione. Per una rilettura dei documenti in forma di lista nei comuni italiani della prima metà del XIII secolo, in I. Lazzarini, G. Gardoni (a cura di), Notariato e medievistica. Per i cento anni di Studi e ricerche di diplomatica comunale di Pietro Torelli. Atti delle giornate di studi (Mantova 2-3 dicembre 2011), Roma, 2013, p. 109-146.

Vallerani 2014 = M. Vallerani, Fiscalità e limiti dell'appartenenza alla città in età comunale, Bologna fra due e trecento, in Quaderni Storici, 147/3, 2014, p. 709-742.

Varanini 1984 = G. M. Varanini, Un quaternus expensarum del comune di Verona (novembre 1279), in Studi di Storia Medioevale e di Diplomatica, 8, 1984, p. 74-100.

Vasina 1965 = A. Vasina, I Romagnoli fra autonomie cittadine e accentramento papale nell'età di Dante, Firenze, 1965.

Vendittelli 2015 = G. Vendittelli, Hoc est memoriale. Due inventari di beni del comune di Bologna negli anni cinquanta del XIII secolo, in Bullettino dell'Istituto Storico Italiano per il Medio Evo, 117, 2015, p. 223-288.

Vitale 1901 = V. Vitale, Il dominio della parte guelfa in Bologna (1280-1327), Bologna, 1901. (ed. anastatica Bologna 1978).

Wray 2009 = S. K. Wray, Communities and crisis : Bologna during the Black Death, Boston, 2009.

\section{Notes}

1 Per una bibliografia aggiornata di Bologna si veda : Storia di Bologna 2007 ; Wray 2009 ; Blanshei 2010 ; Milani 2012. Per gli studi sulla fiscalità citati : Livi 1933 ; Sella 1933.

2 Orlandelli 1951 ; Orlandelli 1954, p. 7-48.

3 Bocchi 1973 ; Castagnini 1976 ; Pini 1977 ; Bocchi 1981 ; Micheletti 1981 ; Frescura Nepoti 1982 ; Giansante 1985 ; Bocchi 1987 ; Foschi 1989.

4 Smurra 2003 ; Smurra 2007 ; Vallerani 2009 ; Vallerani 2014 ; Vendittelli 2015 ; Milani 2015.

5 Ginatempo 2000 ; Ginatempo 2013.

6 ASBo, Comune, Camera del Comune (sec. XIII-sec. XVI), Tesoreria e contrallatore di tesoreria 1254-1524, Liber expensarum $n^{\circ} 3$. (Indicato da questo momento come, ASBo, Liber expensarum $n^{\circ} 3$ ).

7 Nello stesso 1288 a Bologna furono promulgati i nuovi statuti cittadini, il cui studio condotto in parallelo a quello dei registri contabili, potrebbe permettere di valutare insieme normativa e prassi in materia di spesa pubblica (Statuti del comune 1869-1884 ; Statuti di Bologna 1939 ; Trombetti Budriesi 2014.

8 ASBo, Comune, Curia del Podestà, Ufficio al Disco dell'Orso, Busta 6 (XIII-XIV sec.) e Busta 16 (XIII-XIV sec.) ; ASBo, Comune, Camera, Soprastanti, depositari e conduttori dei dazi, Busta 114 (1272-1292). Un frammento dell'officium bladi si trova nel fondo Abbodanza e della Grascia, che è attualmente fuori consultazione.

9 W. M. Bowsky 1975 ; P. Mainoni 2007, p. 358.

10 Per il controllo si veda : Orlandelli 1954 ; per la parte relativa la conservazione si veda : Romiti 1994.

11 ASBo, Comune, Camera del Comune (sec. XIII- sec. XVI), Tesoreria e contrallatore di tesoreria 1254-1524, Liber introituum $1298 n^{\circ} 4$ bis.

12 Romiti 1994, p. 307.

13 Statuti di Bologna 1939, vol. I, p. 79-80.

14 ASBo, Comune, Camera del Comune (sec. XIII-sec. XVI), Tesoreria e contrallatore di tesoreria 1254-1524, Liber introituum $1298 n^{\circ} 4$ bis ; Liber introituum $1308 n^{\circ} 6$; et Liber introituum 1309-1310 $n^{\circ} 7$.

15 A proposito si veda : Orlandelli 1954.

16 ASBo, Comune, Camera del Comune (sec. XIII-sec. XVI), Tesoreria e contrallatore di tesoreria 1254-1524, Liber expensarum $1288 n^{\circ} 3$ et Liber expensarum $1296 n^{\circ} 4 a$. Per il 
liber expensarum del 1297, si tratta di un registro mutilo, erroneamente conservato nella serie Pagamenti degli stipendiari (ASBo, Comune, Camera del Comune, Miscellanea, Pagamenti degli stipendiari 1297-1525).

17 Su queste questioni la letteratura è molto ampia, si veda : Albini 1988 ; Baietto 2000 ; Lazzarini 2001 ; Baietto 2002 ; Bartoli Langeli 2006 ; Comptabilités pontificales 2006 ; Grillo 2006 ; Approche codicologique 2011.

18 ASBo, Liber expensarum $n^{\circ} 3$, c. $1 \mathrm{r}$.

$19 \mathrm{Si}$ tratta di un notaio piuttosto attivo in quegli anni : nel 1288 scrive diverse riformagioni per il consiglio degli Ottocento ; nel 1302-1303 è notaio ad acta comunis Bononie; e nel 1317 fu proconsole della società dei notai. (Società dei notai 1988, p. 205 e 306 ;

20 Proprio questo tipo di aggiunte dovevano essere l'oggetto del seguente passo degli Statuti del 1288 : "Millesimo ducentesimo nonagesimo, inditione tercia, die penultima decembris. Reformatum fuit tempore domini Fulchi de Buçacarinis capitanei populi Bononie quod massarii et depositarii generales comunis Bononie et eorum notarii modo electi, et qui pro tempore erunt ad dicta ofitia exercenda, teneantur et debeant scribere introitus et expensas distinte et continue per capitula, non inserendo alios introitus vel expensas nisi que continebuntur sub dicti capitulis, videlicet capitulum denariorum receptorum de gabelle et capitulum denariorum receptorum ad dischi Ursi, et sicut de singulis, de omnibus introitibus et expensis ordinariis comunis Bononie, sub certa pena et banno cuilibet eorum » (Statuti di Bologna 1939, vol. I, p. 115).

21 Altri registri contabili prodotti dalla tesoreria della metà-fine del XIII secolo sono conservati nell'Archivio di Stato di Bologna (1254, 1255, 1295, 1296, 1298 e 1299). Tutti questi documenti hanno una taglia media di mm 443×309. (ASBo, Comune, Camera del Comune (sec. XIII-sec. XVI), Tesoreria e contrallatore di tesoreria 1254-1524, Registri $n^{\circ} 1-6$ (1254-1308).

22 Non siamo in grado di capire quante carte sono andate perdute, visto che non si vedono i talloni delle carte, e non conosciamo la quantità precisa dei pagamenti registrati tra le due date. 23 ASBo, Comune, Ufficio dei Fortilizi e delle Munizioni dei Castelli, Busta 5, Liber expensarum 1288

24 Elementi che saranno descritti in seguito (corrisponde la datazione, la numerazione dei pagamenti, la dimensione delle carte, il supporto, la scrittura del notaio, gli elementi di contabilità, ecc.).

25 ASBo, Comune, Ufficio dei Fortilizi e delle Munizioni dei Castelli, Busta 5, Liber expensarum 1288; I libri securitatum et actorum del 1285, 1287, 1288 hanno un supporto cartaceo, invece quelli del 1290, 1291 sono in pergamena, come tutti i libri contractuum di quegli anni (Orlandelli 1954, p. 5-13). Riguardo il supporto va notato che tutti gli altri registri di entrate e uscite sono in pergamena fino alla fine del XIII secolo. Si tratta di dati che indicano una pratica documentaria ancora non uniforme, come sembra confermare l'assenza di indicazioni al riguardo negli Statuti del 1288. Come abbiamo visto in precedenza, è possibile quindi che la scelta del supporto sia legata alla funzione preparatoria del registro.

26 ASBo, Comune, Camera del Comune (sec. XIII-sec. XVI), Tesoreria e contrallatore di tesoreria 1254-1524, Liber expensarum $1296 n^{\circ}$ 4, e Liber expensarum $1303 n^{\circ} 5$.

27 Come scrive Massimo Vallerani questo elemento è stato un prodotto dei conflitti che sin dal primo periodo podestarile « innescarono un meccanismo a catena che costrinse gli ufficiali del comune a elaborare sempre nuovi strumenti di definizione della cittadinanza e di ripartizione controllata delle risorse ». Per esempio : « la singola informazione doveva essere non solo riprodotta nella sua completezza contabile, ma contenere anche gli estremi della sua legittimazione politica : chi aveva autorizzato la spesa, chi aveva convalidato quell'atto, quale notaio aveva scritto la carta e così via ». (Vallerani 2013, p. 119).

28 Con la frase salvo erori calcholi terminano le registrazioni del secondo semestre del 1288 , dopo il breve sommario delle uscite totali posto nell'ultima carta del registro. La frase, che appartiene al formulario della documentazione amministrativa, mette in guardia da eventuali errori che effettivamente è possibile riscontrare, in particolare nel calcolo della somma totale di ogni singolo mese, ma che rimangono comunque molto limitati. Per esempio, nel caso del 
resoconto del mese di ottobre l'errore sembra dovuto ad un errato incolonnamento delle varie monete da parte del notaio al momento del calcolo. Il notaio indica 20 lire in più, al posto di un soldo, scambiando il soldo con la lira. (ASBo, Liber expensarum $n^{\circ} 3$, c. $111 \mathrm{v}$ ).

29 Il comune di Milano sembra avere la stessa attenzione per il controllo contabile : Grillo 2001, p. 533.

30 Varanini 1984.

31 Acta Comunitatis Tarvisii 1998.

32 Palmieri 1889, p. XVIII-XXVIII.

33 Ibid., p. XXVIII.

34 I valori (espressi in denari) sono i seguenti :

35 Statuti di Bologna 1939, vol. II, p. 187 ; (Rubrica pubblicata anche in Bordone 1984).

36 Ibid., vol. II, p. 187-188.

37 Ibid., vol. II, p. 188.

38 Ibid., vol. II, p. 196. Sui risarcimenti ai cavalieri si veda : Maire Vigueur 2004 ; Lazzari 2011 ; Grillo 2008, p. 137-138.

39 Altre informazioni sulla spesa pubblica del comune prima del 1288 sono presenti in Hessel 1975 ; Milani 2015. Riguardo la fine del XIII secolo, nel secondo semestre del 1270 le entrate furono di 72.347 lire e le uscite di 72.032 lire, invece per i primi sei mesi del 1271 le prime risultano 41.915 lire e le seconde 41.925 lire. Affiancando questi ultimi due dati alle uscite del secondo semestre del 1270, ammontanti a 72.032 lire, si può subito notare che siamo in un ordine di grandezza quasi del doppio dei dati sopraccitati. Questa grande differenza va senza dubbio spiegata con l'inizio del conflitto tra Bologna e Venezia nella tarda estate del 1270. Gli attriti tra queste due città cominciarono nel 1257, quando Bologna dopo una richiesta di aiuto da parte di Ravenna tentò di sottometterla, quindi inserendosi nell'area di dominio veneziano. Venezia spronò anche Cervia e Ravenna a non rispettare i loro accordi con la città, per farle entrare nel suo emisfero d'influenza. Il conflitto armato arrivò, secondo Hessel, solo perché Venezia per esigenze proprie bloccò tutti gli approvvigionamenti dal mare. Altri dati sembrano confermare l'ipotesi visto che, proprio nel 1270 il comune si fece prestare 5.000 lire per l'acquisto di viveri e nell'autunno seguente chiese un prestito forzoso di 33.000 lire.

40 ASBo, Liber expensarum $n^{\circ} 3$, c. 96 r.

$41 \mathrm{Ibid}$., c. 101v.

42 Ibid., c. 108v.

43 In un altro pagamento di quattro giorni precedenti al suddetto si pagano 50 lire per il prestito sopra citato, quindi è presumibile che si tratti dell'interesse, modesto, dato che ammontava al $2,5 \%$. Questo prestito al comune rimane l'unico registrato in tutto il documento. ASBo, Liber expensarum $n^{\circ} 3$, c. $104 \mathrm{r}$.

44 ASBo, Liber expensarum $n^{\circ} 3$, c. 37r.

45 Ibid., c. 66 r.

46 Ibid., c. $52 \mathrm{v}$.

47 Ibid., c. 46v.

48 Ibid., cc. $28 \mathrm{v}$ e $78 \mathrm{v}$.

49 Ibid., c. 1v; Statuti di Bologna 1939, vol. I, p. 103-105 ; Tamba 1982 ; Democrazia diretta 1997. L'elezione ad brevia era una procedura piuttosto comune all'epoca. Gli elettori dopo aver scritto sul brevia, un pezzo di pergamena, il nome della persona a cui affidare l'incarico, lo riponevano in un sacchetto. Dal sacchetto poi sarebbero stati estratti tutti i voti e conteggiati. Questo sistema permetteva di limitare la formazione di gruppi troppo forti all'interno delle strutture cittadine. Per la loro estrazione si pagano numerose volte dei bambini, 1 soldo a testa, « qui dederunt brevia ex quibus ellecti fuerunt capitaneus et custodes castrorum ». Come attestano gli Statuti del 1288 questi custodi e capitani dovevano avere alcuni requisiti per poter essere eletti : «non possit aliquis elligi ad custodiam...nisi sit de societate artium et armorum tantum, cambi et mercandadie, et sit et fuerit continuus habitator civitatis Bononie vel burgorum per viginti annos et habeat extimum in comuni saltim vigintiquinque librarum bononinorum ».

$50 \mathrm{ASB}$, Liber expensarum $n^{\circ} 3$, cc. $78 \mathrm{r}-79 \mathrm{v}$. 
51 Ibid., cc. $52 \mathrm{v}-64 \mathrm{v}$.

52 Ibid., cc. $101 \mathrm{v}$ e $102 \mathrm{r}$.

53 Ibid., c. 49v.

54 Ibid., c. 102 r.

55 Ibid., cc. 96r-98v.

56 Ibid., cc. 22r, 22v, 23v, 26v, 27r, 91v e 95v. Il registro inoltre fornisce informazioni anche sulla durata di altre cariche e sui tempi dei pagamenti, che appaiono di solito sistematicamente sfasati rispetto al tempo effettivo dei lavori o servizi eseguiti. (Talvolta i pagamenti sono anticipati, altre volte vengono compiuti a servizio in corso, come avviene giovedì 23 dicembre quando si pagano dei mercenari, i quali avevano iniziato a servire il comune il 13 novembre. Numerosi sono i pagamenti fatti a breve distanza dal lavoro o servizio eseguito, ad esempio, il giorno 5 luglio viene fatto un pagamento " pueris qui dederunt brevia ex quibus ellecti fuerunt capitaneus et custodes castrorum et quidam alii officiales in conscilio duorum millium die quarto iullii ». Alcuni invece sono fatti con maggiore ritardo. In data 6 luglio, il comune paga «Iohannino Symonis nuntio ançianorum et consulum populi qui expensavit de suis propriis denariis in cartis bombicinis et in medio quaterno cartarum peccudum operatis in servitio comunis quando facta fuit ellectio capitanei populi de mense iunii preterito ». In molti altri casi non ci sono indizi per ricavare informazioni utili ai tempi di pagamento. Si fa riferimento al servizio eseguito senza aggiungere nulla sui tempi, come avviene per un lavoro effettuato da Deomede Raymundini. Informazioni presenti in ASBo, Liber expensarum $n^{\circ} 3$, cc.1v, 2r, 8r e 102r.)

57 Ibid., cc. 79v-80v. Greci - Pini 1976.

58 Ibid., c. $56 \mathrm{v}$.

59 Pinto 1987 ; Giansante 1985-1986 ; Disette dans la conjoncture 2011 ; Magni 2015.

60 ASBo, Liber expensarum $n^{\circ} 3$, c. $12 \mathrm{v}$.

61 Ibid., c. 5r.

62 Ibid., c. 12r ; Vasina 1965.

63 Ibid., c.106r.

64 Ibid., cc. 40v, 41r e 108v.

65 Ibid., c. 40v.

66 Ibid., cc. 88 r e $106 r$.

67 Ibid., c. 11 r.

68 Ibid., cc. $37 \mathrm{v}, 54 \mathrm{v}$ e $82 \mathrm{v}$.

69 Per informazioni sui due pittori si veda : Filippini - Zucchini 1947 p. 48-53 ; Cova 2010.

70 ASBo, Liber expensarum $n^{\circ} 3$, c.107r.

71 Statuti di Bologna 1939, vol. I, p. 308-312.

72 A proposito della pittura infamante si veda : Ortalli 1979.

73 ASBo, Liber expensarum $n^{\circ} 3$, cc. $26 \mathrm{v}, 34 \mathrm{v}$ e $59 \mathrm{v}$; Forse si trattava di nobili, poiché una provvigione del 1287 stabiliva 50 lire di ricompensa per la cattura di un nobile bandito e 25 lire per un popolano (Vitale 1901, p. 41).

74 ASBo, Liber expensarum $n^{\circ} 3$, c. 32r ; Per comprendere meglio il pagamento suddetto bisogna vedere gli Statuti del 1288, i quali a proposito dicono : «Ordinamus quod aliqua persona non teneat aliquam porcham cum filiis in civitate Bononie vel sine filiis in burgis vel iuxta civitatem Bononie per unum miliare » " item dicimus quos nullus porchus vel porcha deberent intrare terminos platee comunis Bononie vel porte Ravenatis et quod notarius domini potestatis inquirere teneatur in qualibet edomada de predictis et si quos invenerint ibi, conduci faciat ad palatium comuni set condempnare eum cuius porchus erit in viginti solidis bononinorum et quolibet possit accusare et denuntiare et habeat mediatatem banni » (Statuti del Comune dell'anno 1288, vol. II, p. 136-137).

75 ASBo, Liber expensarum $n^{\circ} 3$, cc. 22r e 25v; A proposito del significato dei leoni ne ha discusso Knut Goerich al convegno : «Enrico VII, Dante e Pisa. A settecento anni dalla morte dell'imperatore e dalla Monarchia » tenutosi a Pisa il 24-26 ottobre 2013 (con un intervento Sulla concezione e rappresentazione del potere di Enrico VII).

76 ASBo, Liber expensarum $n^{\circ} 3$, c. 46v. 
77 Ibid., cc. $3 \mathrm{r}$ e $10 \mathrm{v}$.

78 Ibid., cc. $23 \mathrm{v}$ e $24 \mathrm{v}$.

79 Vitale 1901, p. 42-43 ; Mentre per l'interesse del comune verso i beni dei Lambertazzi si veda : Milani 2003, p. 315-364.

80 Si parla solo di quatuor notariis deputatis ad officio malloficiorum e « dictis aliis notariis [...] qui sunt numero .XXXIIII. », quindi 38 notai, ma nel documento sono segnati 40 notai, e mancano all'appello proprio i notai al disco della volpe, e del resto neanche contati nell'intestazione della spesa, che prevede come detto 4 lire per i notai ad officio malleficiorum e 3 lire per altri 34 notai, e appunto dalla spesa risulta 118 lire per sei mesi di servizio. Si veda : ASBo, Liber expensarum $n^{\circ}$ 3, c. 70r ; Statuti di Bologna 1939, vol. II, p. 188.

81 ASBo, Liber expensarum $n^{\circ} 3$, cc. 19r e 73r.

82 Per i salari degli ufficiali della città, negli Statuti del 1288 esiste una rubrica specifica «De expensis ordinariis faciendis per comune Bononie »; Statuti di Bologna 1939, vol. II, p. 187-190 ; si veda anche la nota ${ }^{\circ} 31$.

83 ASBo, Liber expensarum $n^{\circ} 3$, cc. 38v e 46v.

84 Vallerani 2005, p. 120.

85 ASBo, Liber expensarum $n^{\circ} 3$, c. $29 \mathrm{v}$.

86 Dai pagamenti è possibile capire che : il pane veniva distribuito ai carcerati una volta al giorno ; il suo costo era di 2 denari al pezzo ; e che l'acquisto del pane veniva eseguito solitamente ogni 3-5 giorni. Inoltre sappiamo che il 3 e 5 luglio si registrano 54 prigionieri nelle carceri comunali, e da quel punto in poi per tutti i mesi successivi risultano tenute in custodia 46 persone (ASBo, Liber expensarum $n^{\circ} 3$, cc. 1v, 2r e 4v).

87 Ibid., cc. 2r e 76v.

88 Il comune inviava in alcune festività i suoi ufficiali alle processioni, chiaramente si dice : « in primis teneatur ire dominus postestas et dominus capitaneus cum eorum familiis et omnibus ancianis et consulibus populi et offitiales curie personaliter annuatim ad festum beati Gregorii [...] cum baldachinis et ceris, in quibus expendantur de avere comunis Bononie L libras bononinorum » (Statuti di Bologna 1939, vol. II, p. 190).

89 Statuti di Bologna 1939, vol. II, p. 190-191 ; ASBo, Liber expensarum n 3, c. 105r. Per le altre festività è impossibile la verifica poiché cadono nel primo semestre dell' anno.

90 La bibliografia sull'argomento è davvero molto ricca, come inizio si vedano i recenti : Grillo 2007 ; Grillo 2008 ; Cittadini in armi 2011.

91 A proposito si veda la nota $\mathrm{n}^{\circ} 57$.

92 Ad esempio si veda, ASBo, Liber expensarum $n^{\circ} 3$, cc. 11v, 100r e 108r.

93 Questa nostra stima è molto vicina a quella fatta in : Tamba 1982.

94 Pini 1996, p. 38-103 ; Precisamente sono 5.325 per il 1273, e 5.271 per il 1306.

95 Il consiglio è stato oggetto di un articolo di Giorgio Tamba, si veda : Tamba 1982. Una delle prime attestazioni del consiglio dei Duemila è del 2 dicembre 1285, giorno in cui furono emanate una serie di norme, tra le quali una che disciplinava l'elezione degli ufficiali di estrazione cittadina per il consiglio dei Duemila. Le origini di questo consiglio sono non del tutto chiare visto una possibile coabitazione o momentanea sostituzione con il consiglio dei Quattromila. Il consiglio dei Duemila fu uno strumento di unificazione dell'amministrazione cittadina, dato che il consiglio eleggeva sia cariche della parte "comunale" che "popolare". Un consiglio che moltiplicava le occasioni di un reale contatto di tutti i cittadini con l'organizzazione cittadina.

96 Il notaio degli anziani e dei consoli del mese di dicembre, Riguçio di Pace, venne pagato il 27 dello stesso mese per il salario mensile 6 lire, e il medesimo giorno ricevette 12 lire per la scrittura di alcune provvigioni, questo a dimostrazione degli ulteriori introiti che potevano riguardare una carica elettiva del comune. ASBo, Liber expensarum $n^{\circ} 3$, c. $24 \mathrm{v}$.

97 Ibid., c. $51 \mathrm{v}$.

98 Ibid., c. $42 \mathrm{v}$.

99 Ibid., cc. $5 \mathrm{r}-7 \mathrm{v}$.

100 Statuti di Bologna 1939, vol. II, p. 217.

101 ASBo, Liber expensarum $n^{\circ} 3$, cc. 88r e 107r. 
102 Ibid., c. 34v.

103 Riguardo al provvedimento si veda : Vitale 1901, p. 40 ; Milani 2003, p. 315-364.

104 Disette dans la conjoncture 2011

105 Per i dettagli si veda la nota ${ }^{\circ} 35$.

106 Knapton 1997 ; Knapton 1998 ; Ginatempo 2000 ; Politiche finanziarie e fiscali 2001 ; Chittolini 2002 ; Lazzarini 2003 ; Milani 2010.

\section{Pour citer cet article}

Référence électronique

Marco Conti, «La spesa pubblica bolognese alla fine del XIII secolo. Prime indagini sul Liber expensarum del $1288 »$, Mélanges de l'École française de Rome - Moyen Âge [En ligne],

128-2 | 2016, mis en ligne le 06 septembre 2016, consulté le 07 septembre 2016. URL : http:// mefrm.revues.org/3329

\section{À propos de l'auteur}

Marco Conti

Université Lumière Lyon 2 - CIHAM / Università La Sapienza di Roma -

\section{Droits d'auteur}

(C) École française de Rome

\section{Résumés}

Il presente articolo vuole analizzare una fonte inedita conservata all'Archivio di Stato di Bologna, il Liber expensarum del 1288. Nella prima parte del contributo sono state studiate le caratteristiche intrinseche ed estrinseche del documento, che ci hanno permesso di stabilire il suo ruolo tra le scritture contabili del comune. Nella seconda parte invece è stato ricostruito il bilancio del secondo semestre del 1288, e sono state analizzate tutte le voci di spesa pubblica del Liber, allo scopo di comprendere la politica fiscale del comune.

This contribution wants to investigate a unpublished source preserved in the Archivio di Stato of Bologna, the Liber expensarum of 1288. In the first part of the contribution were studied the intrinsics and extrinsics characteristics of the document, that give us the possibility to determinate his role between the accounting records of the commune of Bologna. In the second part, the budget of the second half of 1288 was rebuilt and it was studied all items of the Liber to understand the fiscal policy of the town.

\section{Entrées d'index}

Keywords : Bologna; Italian city-states, fiscal policy, accounting records Parole chiave : Bologna, comuni, politica fiscale, scritture contabili 